\title{
A REVIEW ON PLC MODELING TECHNIQUES FOR RESIDENTIAL NETWORKS
}

\author{
Shashidhar Kasthala \\ Faculty of ECE \\ Indian Naval Academy \\ Ezhimala, India
}

\author{
G.K.D. Prasanna Venkatesan \\ Research and Development Cell \\ SNS College of Engineering \\ Coimbatore, India
}

\begin{abstract}
This paper discuss about the recent developments in the field of power line communication for residential networks. In the recent past, various techniques have been developed to model the power line channel. However, none of these techniques could realize a power line channel accurately. Measurement campaigns were also undertaken across the world to understand the dynamic behavior and statistically analyze the power line channel. The major complexity in PLC is the difference in the power line characteristics across the world.
\end{abstract}

Keywords: Power line communication, Channel transfer function, Stationary Noise, Impulsive Noise

\section{INTRODUCTION}

With the rapid development in the network technology, home automation and in-home network connectivity as attracted both the industry and scientific community. Though various communication technologies are being tried out for this, power line communication (PLC) has its own advantages [1]. Since no additional infrastructure is required, PLC can be an economical option. The deep coverage of electrical wires to every part of the building is another advantage. Apart from in-home networks, PLC is also being tried out on medium voltage networks, low voltage networks and in-vehicle networks.

Communication over residential power line can be carried out in two different frequency bands. These bands are generally called as narrowband PLC and broadband PLC. Narrowband PLC in 1-500 kHz frequency band can be used for home automation and security applications [2][3]. Broadband PLC in the frequency band of 1-200 MHz can be used for HDTV, high speed internet access and gaming activities. In fact, a data rate of $200 \mathrm{Mbps}$ can easily be achieved through broadband PLC.

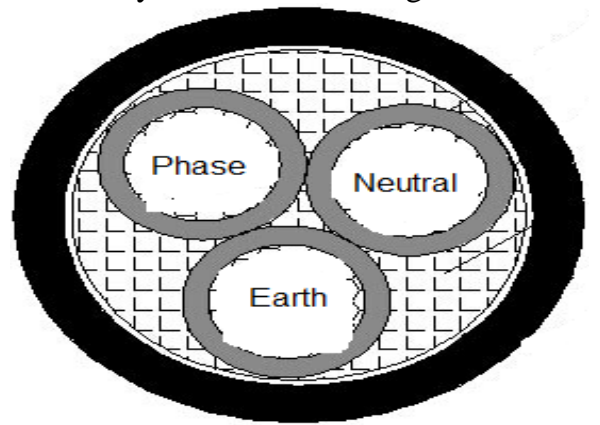

Figure 1. Residential Wiring

The residential electrical network contains three wires viz. Phase Neutral and Earth. Communication over power line can be carried out using and two wires of this network, preferably Phase and Neutral. Using two wires is usually sufficient for home automation and medium speed requirement applications. However, PLC can also achieve high speed by making use of all the three wire of the network [4][5]. This is termed as MIMO power line communication where in all the three wires are used simultaneously to transmit and receive the data. In fact, an additional extra port can also be created for receiving the data called as common mode port. This port is created due to the unbalanced currents in the network. MIMO PLC has attracted many researchers similar to MIMO wireless communication [6][7].

Apart from the various advantages of the PLC, there are certain challenges with this technology. The foremost is that the power lines were never designed for communication and is inherently hostile to data communication. The other challenges are signal attenuation, impedance mismatch and the noise in the power line.

The loads connected to the power line do not operate continuously and this leads to constant impedance mismatch between the transmitter and the receiver. This variation in load will also affect the signal transmission since the attenuation is due to the transmission and reflections in the power line and branches. The noise in the power line is very unpredictable and can be due to the loads connected.

The noise in the power line can be broadly classified into stationary noise and impulsive noise. The stationary noise is the inherent noise present in the power line and does not vary with time. But the impulsive noise is due to the loads connected and will dynamically vary with time.

To develop a reliable communication system over power lines, it is very important to understand these characteristics and develop appropriate modulation techniques to counter the data loss in the channel. Advanced modulation techniques like OFDM and MCDMA are being worked out for the same [8][9][10][11][12][13]. In this paper, the work being carried out in the field of power line communication on residential networks is discussed in detail.

The subsequent sections of this paper are organized as follows. In the section II, the various channel modeling techniques developed so far in modeling the residential power line is discussed. In the section III, the measurement campaign undertaken across the world to statistically analyze the power line channel is discussed.

\section{Channel Modeling Techniques}

For indoor power line communications, various channel modeling techniques have been developed [14]. These modeling techniques are either based on the multipath propagation or on the transmission line theory. The former is a 
straight forward technique and is based on parameters, whereas the latter is based on the information obtained from the network.

The commonly used multipath propagation model is expressed as

$$
H(f)=\sum_{i=1}^{N} g_{i} e^{-\left(a_{0}+a_{1} f^{k}\right) \cdot d_{i}} e^{-j 2 \pi f \frac{d_{i}}{v_{p}}}
$$

Here, for $\mathrm{N}$ number of paths, gi is the weighing factor obtained from the transmission and reflection coefficients and di is the length of the branch. a0, a1 and $\mathrm{K}$ are the attenuation constants. The sample network to explain a multipath propagation is shown in Figure. 2.

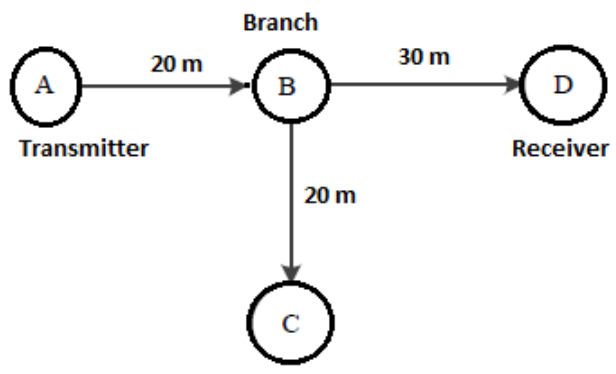

Figure 2. Sample network

In this work the typical PLC channel with 6 paths is considered because of the fact that longer paths have higher attenuation and they contribute less to overall signal at the receiving point. The parameters of cable considered for the path is listed in Table I.

Table I. Parameters of sample Network

\begin{tabular}{|c|c|c|}
\hline Path No. (i) & Length (di) & $\begin{array}{c}\text { Weighting } \\
\text { factor (gi) }\end{array}$ \\
\hline 1 & 200 & $0 . .54$ \\
\hline 2 & 221 & 0.275 \\
\hline 3 & 242 & -0.15 \\
\hline 4 & 259 & 0.08 \\
\hline 5 & 266 & -0.03 \\
\hline 6 & 530 & -0.02 \\
\hline
\end{tabular}

The amplitude response of the sample network is shown in Figure 3. The attenuation in the signal can be inferred from the peaks and valleys of the amplitude response.

Though this approach is very simple, it cannot reflect the power line channel accurately. The reason for this is that the approach is based on parameters and these parameters may not take into account every characteristics of the power line.

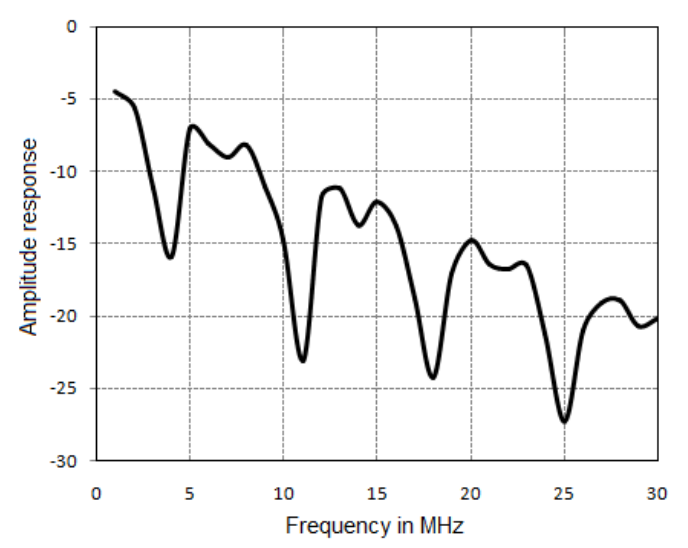

Figure 3. Amplitude response for multipath approach
Another approach of modeling the channel is based on the transmission line theory. This is usually carried out in frequency domain. The transmission line can be represented as a series of two port networks cascaded together as shown in Figure. 4.

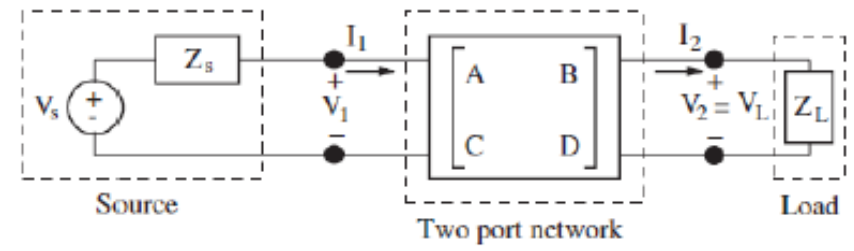

Figure 4. Two port Networks

These two ports networks can be represented using ABCD parameters and all the networks can be cascaded together to form the entire network. The channel transfer function can be calculated from the relationship between input and output voltages and currents.

The relation between the voltage and currents for a two port network can be represented as

$$
\left[\begin{array}{l}
\mathrm{V}_{1} \\
\mathrm{I}_{1}
\end{array}\right]=\left[\begin{array}{ll}
\mathrm{A} & \mathrm{B} \\
\mathrm{C} & \mathrm{D}
\end{array}\right]\left[\begin{array}{l}
\mathrm{V}_{2} \\
\mathrm{I}_{2}
\end{array}\right]
$$

The transfer function of the network using ABCD matrices can be expressed as

$$
H(f)=\frac{Z_{L}}{A Z_{L}+B+C Z_{S} Z_{L}+D Z_{S}}
$$

The ABCD matrix of a transmission line can be represented in terms of impedance and propagation constant. These are represented as

$$
\gamma=\sqrt{\left(R+\sqrt{\frac{R+J w L}{G+j W C}}\right.}
$$

The ABCD matrix can be represented as

$$
\left[\begin{array}{ll}
A & B \\
C & D
\end{array}\right]=\left[\begin{array}{cc}
\cosh (\gamma L) & Z \sinh (\gamma L) \\
\frac{1}{Z} \sinh (\gamma L) & \cosh (\gamma L)
\end{array}\right]
$$

The other methods in this approach are based on the voltage ratio approach and $\mathrm{S}$ parameter approach. The $\mathrm{S}$ parameter method can also be carried out in wavelet transformation methods. The S matrix representation of the network is shown as

$$
\mathrm{S}=\left[\begin{array}{ll}
\mathrm{S}_{11} & \mathrm{~S}_{12} \\
\mathrm{~S}_{21} & \mathrm{~S}_{22}
\end{array}\right]=\left[\begin{array}{cc}
\frac{\mathrm{T}_{21}}{\mathrm{~T}_{11}} & \mathrm{~T}_{22}-\frac{\mathrm{T}_{21} \mathrm{~T}_{12}}{\mathrm{~T}_{11}} \\
\frac{1}{\mathrm{~T}_{11}} & -\frac{\mathrm{T}_{12}}{\mathrm{~T}_{11}}
\end{array}\right]
$$

The transfer function obtained for this method will be

$$
H(f)=\frac{z_{L}}{T_{11} Z_{L}+T_{12}+T_{21} z_{s} z_{L}+T_{22} z_{s}}
$$

This approach is though comparatively complex than the previous approach, it reflects the power line channel to larger extent and is independent of the number of branches and complexity of the network. 


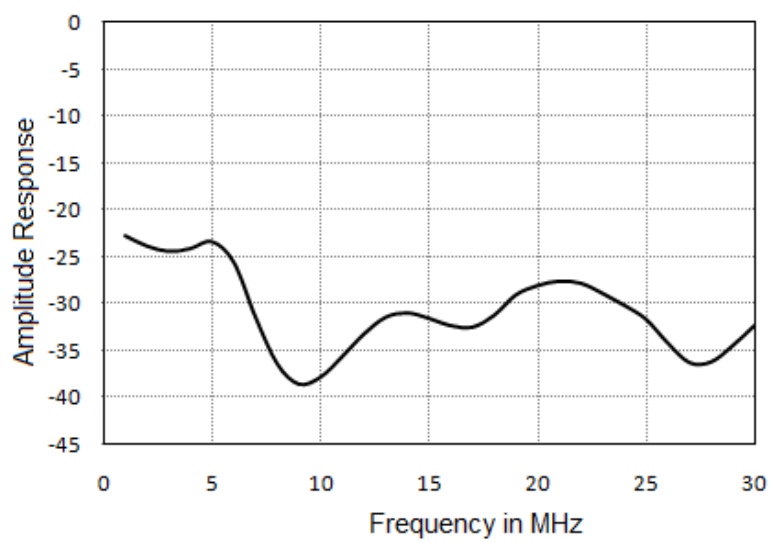

Figure 5. Amplitude response for transmission matrix

However, these two traditional approaches are yet to realize the complete power line channel. The major challenges in the power line medium, unlike any other communication medium, are the dynamical variation in the load conditions, affect of the ambient conditions [15]. To ascertain the behavior of the channel in the modeled approach, researchers prefer statistical analysis.

\section{Measurment Campaign}

To perform the statistical analysis on the power line, it is mandatory to build an appropriate experimental setup. The most important component for this is to build a coupling interface. The objective of this coupling interface is to isolate the measuring equipment from the high voltage power supply and also measure the characteristics in the required bandwidth [16].

The coupling circuit should also be provided with protective devices so as to avoid any lighting strikes and surges damaging the equipment. The indoor residential networks are prone to the surges caused due to the switching of inductive devices.

Measurement campaigns are conducted across the world, particularly in Europe to evaluate the power line channel. The parameters under consideration were channel transfer function, stationary noise and impulsive noise.

To characterize the channel transfer function, the statistics of the amplitude of the frequency response in studied in detailed. For this, the path loss and the probability density function are estimated. The path loss can be represented as

$$
\mathrm{L}(\mathrm{n})=10 \cdot \log _{10}|\mathrm{H}(\mathrm{n})|^{2}
$$

The channel capacity can be represented as [10]

$$
\mathrm{C}=\Delta \mathrm{f} . \sum_{\mathrm{i}=1}^{\mathrm{N}} \log _{2}\left[1+\frac{\mathrm{P}\left(\mathrm{f}_{\mathrm{n}}\right)\left|\mathrm{H}\left(\mathrm{f}_{\mathrm{n}}\right)\right|^{2}}{\mathrm{~N}\left(\mathrm{f}_{\mathrm{n}}\right)}\right] \mathrm{bits} / \mathrm{s}
$$

With a transmitted power of $-50 \mathrm{dBm}$ and noise PSD of $140 \mathrm{dBm}$, the minimum and maximum channel capacity obtained in European residential channels is 1 Gbps and 2.8 Gbps respectively.

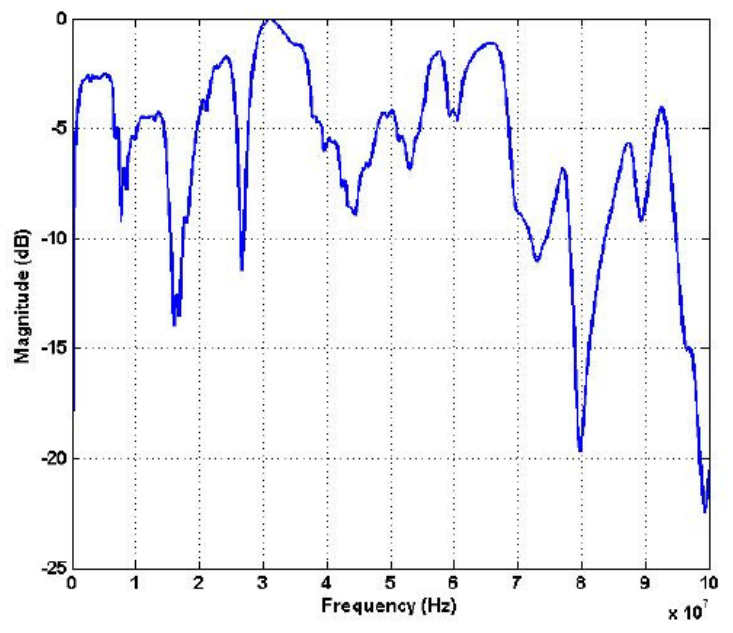

Figure 6. Measured CTF in Italy [17]

Though the stationary noise may be different from country to country due to the difference in load behavior, the noise spectrum nature is almost similar as shown in Figure. 7. At lower frequencies, the noise is noticed to be more and at lower frequencies it is similar.

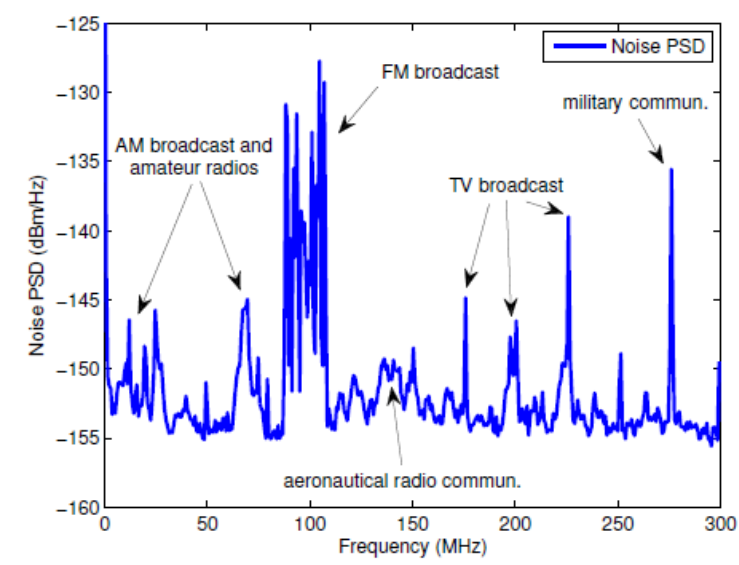

Figure 7. Stationary noise in Italy [17]

The noise is also found to have ingress due to the broadcast signals, which is prevalent between 1-100 MHz.

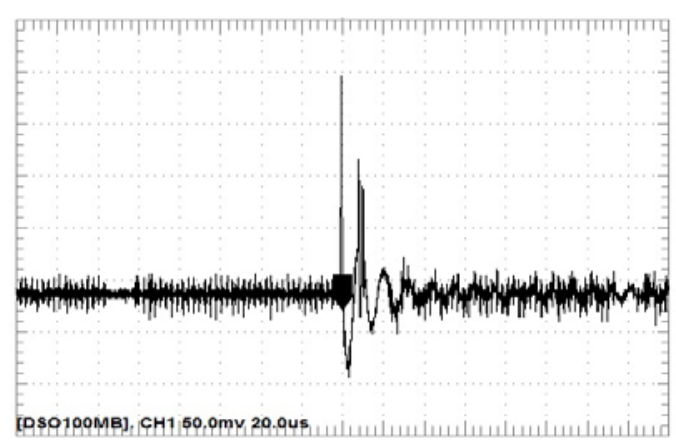

Figure 8. Impulsive noise

The Impulsive noise is in the form of burst with a short duration and can be measured using a Digital Signal Oscilloscope connected to the mains supply via coupling circuit. An impulsive noise of single burst is captured and is as shown in Figure 8. 


\section{CONCLUSIONS}

This paper discusses reviews about the parameters to be considered in characterizing a power line channel. It also presents the work carried out by various organizations on residential networks. To develop an effective PLC system, it is of utmost important to analyze these parameters in every part of the world.

\section{REFERENCES}

[1] Babysen R, Manikandan P and G.K.D.Prasanna Venkatesan, "Various Indoor OFDM Optical Wireless Communication Systems and Performance Characteristics,” Vol. 3, No. 5, 2014.

[2] Krishnapriya, Shashidhar kasthala, "Identification of Cable Faults Onboard Ship using Power Line Communication.," International Journal of Advanced Research in Computer Science, Vol. 8, No.3, pp. 70-73.

[3] S. Saranya, A.Vijay, G.K.D.Prasanna Venkatesan. ,"A Hybrid Communication Infrastructure Power System Using Effective Sensor Network", International Journal of Research in Engineering and Advanced Technology, Volume 2, Issue 2, AprMay, 2014.

[4] Shashidhar Kasthala, GKD Prasanna Venkatesan, "Estimation of MIMO Power Line Communication Channel Capacity using Multi-Conductor Transmission Line Theory,” IEEE International Conference on Applied and Theoretical Computing and Communication Technology.

[5] Shashidhar Kasthala, GKD Prasanna Venkatesan, A Amudha, "MIMO PLC Channel Modelling on Indian Residential Networks" International Journal of Applied Engineering Research, Vol. 12, No. 14, 2017.

[6] S. Ramya, GKD Prasanna Venkatesan, "Study of various transmission schemes of MIMO systems,” International Journal of Emerging Trends in Engineering and Development, Vol. 2, No. 3, 2013.

[7] Banupriya.R, R.R.Jegan and G.K.D.Prasanna Venkatesan,"Disseminating For Concurrent Wireless Data And Power Transfer Using MIMO",International Journal of Research in Engineering \& Advanced Technology, Vol. 2, No.2, 2014.
[8] V Ravichandran, GKD PrasannaVenkatesan, R Rani, "CDMA coding techniques for interconnect between ip cores,” IOSR Journal of Engineering, Vol. 2, No. 9, 2012, pp. 84-90.

[9] GKD Prasanna Venkatesan, VC Ravichandran, "Performance Analysis of Dynamic Sub-Carrier Allocation Technique for Adaptive Modulation based MC-CDMA System”, International Journal of Computer Science and Network Security, VOL.7 No.2, February 2007, pp. 201-204.

[10] GKD Prasanna Venkatesan, VC Ravichandran, "Performance analysis of MC-CDMA for wide band channels," Information technology journal, VOl 6. No. 2, 2007, pp. 267-270.

[11] J Kirubakaran, GKD Prasanna Venkatesan, "Performance Analysis of MIMO based ASTM-OFDM System for Indoor Communication,” International Journal of Advanced Engineering Technology, Vol. 8, No. 2, 2016, pp. 662-666.

[12] V Ravichandran, GKD Prasanna Venkatesan, "CDMA Technique with Inter-process Communication," Research Journal of Applied Sciences, Engineering and Technology, Vol. 7, No. 8, 2014.

[13] G.K.D.Prasanna Venkatesan J.Kirubakaran, "Performance analysis of MIMO systems using CDMA for 4G Wireless Communication,” International Journal of Applied Engineering Research, Vol 10, No. 41, 2015.

[14] Shashidhar Kasthala, GKD Prasanna Venkatesan, "Evaluation of Channel Modeling Techniques for Indoor Power Line Communication”, International Conference on Advanced Computing and Intelligent Engineering, Bubhaneshwar, Dec 2016.

[15] Shashidhar Kasthala, GKD Prasanna Venkatesan, Experimental Verification of Distributed Parameters on Indian Residential Networks for Power Line Communication,” International Journal of Engineering \& Technology, Vol. 8, No. 6, 2012.

[16] Shashidhar Kasthala, GKD Prasanna Venkatesan, A Amudha, "Design and Development of Protective Coupling Interface for Characterizing the Residential Broadband PLC Channel," Journal of Advanced Research in Dynamical and Control Systems, 9(2), 2017

[17] Fabio Versolatto and Andrea M. Tonello, "PLC Channel Characterization up to $300 \mathrm{MHz}$ :Frequency Response and Line Impedance", IEEE symposium on Selected areas in communciation, 2012, pp. 3526-3530. 